United Nations Educational Scientific and Cultural Organization and

International Atomic Energy Agency

THE ABDUS SALAM INTERNATIONAL CENTRE FOR THEORETICAL PHYSICS

\title{
NONRESONANCE CONDITIONS ON THE POTENTIAL FOR A SEMILINEAR DIRICHLET PROBLEM
}

\author{
Aboubacar Marcos ${ }^{1}$ \\ Université Nationale du Bénin, Institut de Mathématiques et de Sciences Physiques, \\ BP. 613, Porto-Novo, Bénin \\ and \\ The Abdus Salam International Centre for Theoretical Physics, Trieste, Italy.
}

\begin{abstract}
Problem $-u^{\prime \prime}=g(u)+h(x)$ in $[a, b]$ with $u=0$ on $\{a, b\}$ is solved under new nonresonance conditions between the first eigenvalue and the first curve in the Fučik spectrum of the associated linear problem.
\end{abstract}

MIRAMARE - TRIESTE

March 2001

\footnotetext{
${ }^{1}$ E-mail: abmarcos@yahoo.fr
} 


\section{Introduction}

This paper is concerned with the solvability of the following problem

$$
\begin{aligned}
-u^{\prime \prime} & =g(u)+h(x) & \text { in }[a, b] \\
u & =0 \quad\{a, b\} &
\end{aligned}
$$

where $g: \mathbb{R} \longrightarrow \mathbb{R}$ is a continuous function. Solutions to (1) are intended in the generalised sense, i.e. $u$ satisfies (1) and $u^{\prime}$ is absolutely continuous.

Various works in the literature are devoted to nonresonance results for problem (1). One can mention for instance the work in [5] where a general nonlinearity is considered, and more recently the work in [3]. In these papers growth restrictions have been required on both the ratios $g(s) / s$ and $2 G(s) / s^{2}$ to control the interference of the nonlinearity with the eigenvalues or the Fučik spectrum of the associated linear problem. In this paper we give new nonresonance conditions between the first eigenvalue and the first non- trivial curve in the Fučik spectrum, on the potential $G$, and prove existence result for (1) Our result is an improvement of that in [5] in the case of problem (1) and is completely different of that in [3]. However some ideas in our work have been developed from [5]. We also mention that the results in [3] have been stated in higher dimension.

The proof of our main result uses estimates for the time-map of a suitable planar system associated to (1) to produce the needed a-priori bounds and combines topological degree argument to conclude. We conclude the paper by giving an example for which our result applies.

\section{General results}

We start this section by stating the preliminary definitions and general results for nonlinear differential equations.

Throughout the paper, $\lambda_{1}$ will denote the first eigenvalue and $\left(\mu_{+}, \mu_{-}\right)$a point of the first curve in the Fučik spectrum of $-\Delta$ on $H_{0}^{1}(a, b)$.

Consider the following parameterized general nonlinear problem

$$
\begin{aligned}
-u^{\prime \prime} & =\bar{g}(x, u, \gamma)+\gamma h(x) \quad x \in[a, b], \quad \gamma \in[0,1] \\
u & =0 \text { on }\{a, b\} .
\end{aligned}
$$

We assume that $h \in L^{1}(a, b)$ and $\bar{g}:[a, b] \times \mathbb{R} \times[0,1] \longrightarrow \mathbb{R}$ satisfies the following:

(i) sign condition: $s \bar{g}(x, s, \gamma) \geq-c$ for a positive constant $c$, for a.e. $x \in[a, b]$ and $\gamma \in[0,1]$;

(ii) $L^{1}$-Caratheodory condition: $\bar{g}(x, ., \gamma)$ is continuous for a.e. $x \in[a, b], \gamma \in[0,1], \bar{g}(., s, \gamma)$ is measurable for all $s \in \mathbb{R}, \gamma \in[0,1]$, and for each $R>0$, there is $a_{R} \in L^{1}(a, b)$ such that $|\bar{g}(x, s, \gamma)| \leq a_{R}(x)$ for all $|s| \leq R$, a.e. $x \in[a, b], \gamma \in[0,1]$.

Solutions of (1) are intended in the generalised sense, i.e. $u$ satisfies (2) for a.e. $x \in[a, b]$ and $u^{\prime}$ is absolutely continuous. For any solution $u$ of (2), let's set 


\section{Definition 2.1}

$$
\begin{aligned}
& x^{*}=\min \{x \in[a, b], u(x)=\max u\} \\
& x_{*}=\min \{x \in[a, b], u(x)=\min u\} \\
& u^{*}=u\left(x^{*}\right), \quad u_{*}=u\left(x_{*}\right)
\end{aligned}
$$

Definition 2.2 For any $K$ such that $0 \leq K \leq u^{*}$, we set

$$
\begin{aligned}
A_{K} & =\{x \in[a, b], u(x)=K\} \\
\alpha_{K} & =\max \left\{x \in A_{K}, x \leq x^{*}\right\} \\
\beta_{K} & =\min \left\{x \in A_{K}, x \geq x^{*}\right\}
\end{aligned}
$$

Definition 2.3 For any $K$ such that $0 \geq K \geq u_{*}$, we set

$$
\begin{aligned}
B_{K} & =\{x \in[a, b], u(x)=K\} \\
\alpha_{K}^{\prime} & =\max \left\{x \in B_{K}, x \leq x_{*}\right\} \\
\beta_{K}^{\prime} & =\min \left\{x \in B_{K}, x \geq x_{*}\right\}
\end{aligned}
$$

Remark 2.1 We note that $x^{*} \in\left[\alpha_{K}, \beta_{K}\right], \quad x_{*} \in\left[\alpha_{K}^{\prime}, \beta_{K}^{\prime}\right]$ and $\left[\alpha_{K}, \beta_{K}\right] \quad\left[\alpha_{K}^{\prime}, \beta_{K}^{\prime}\right]$ are decreasing sequences intervals with respect to $K$.

In general we have the following

Lemma 2.1 For any $A>0$, there exists a constant $M$ (depending only on $A$ ) such that if $u$ is a solution of (2) for $\gamma \in] 0,1$, such that $u$ changes sign and $\max u<A$ or $\min u>-A$, then $u$ satisfies $\|u\|_{\infty}<M$.

\section{Proof of lemma 2.1}

The proof of this lemma is similar to that stated in [4] for $\bar{g}$ not depending on $x$. So we will just give the main idea here. We will consider the case $\max u<A$. The second case $(\min u>-A)$ is similar. Thus suppose on the contrary that there exist a sequence $\left(\gamma_{n}\right) \in[0,1]$ which we write $(\gamma)$ for simplicity of notation and corresponding solutions $u_{n}$ of (2), with max $u_{n}<A$ and $\min u_{n}$ tending to $-\infty$. From the sign condition on $\bar{g}$ and the $L^{1}$-Carathéodory condition we have

$$
\bar{g}(x, s, \gamma) \leq c \chi_{\left\{u_{n}<0\right\}}+a_{R} \cdot \chi_{\left\{0<u_{n}<A\right\}}=a(x)
$$

where $\chi$ is the characteristic function. Take $\tilde{u}_{n}=u_{n}-\bar{u}_{n}$ with $\bar{u}_{n}=\left(x^{*}-x_{*}\right)^{-1} \int_{x_{*}}-x^{*} u_{n}(x) d x$ where we suppose without loss of generality that $x^{*}>x_{*}$. Then multiplying the first equation in (2) by $\tilde{u}_{n}$, we have

$$
\int_{x_{*}}^{x^{*}}\left(\tilde{u}_{n}^{\prime}(x)\right)^{2} d x=\int_{x_{*}}^{x^{*}}\left[\bar{g}\left(x, u_{n}, \gamma\right)-a(x)\right] \tilde{u}_{n} d x+\int_{x_{*}}^{x^{*}}[\gamma h(x)+a(x)] \tilde{u}_{n} d x
$$




$$
\leq\left\|\tilde{u}_{n}\right\|_{\infty}\left\{\int_{x_{*}}^{x^{*}}\left[-\bar{g}\left(x, u_{n}, \gamma_{n}\right)+a(x)\right] d x+\|h\|_{1}+\|a\|_{1}\right\}
$$

where \|\|$_{\infty}$ denotes the uniform norm on $C[a, b]$. But since

$$
\int_{x_{*}}^{x^{*}}\left[-\bar{g}\left(x, u_{n}, \gamma_{n}\right)+\gamma h(x)\right] d x=0
$$

we have

$$
\int_{x_{*}}^{x^{*}}\left(\tilde{u}_{n}^{\prime}(x)\right)^{2} d x \leq 2 R\left\|\tilde{u}_{n}\right\|_{\infty} \text { with } R=\left[\|h\|_{1}+\|a\|_{1}\right] .
$$

Now, using Sobolev inequality on the set of functions of mean value zero, the right-hand side of the above inequality can be minorated by $\|u\|_{\infty}^{2}$ and thus we get

$\|u\|_{\infty}^{2} \leq 2 R . D\left\|\tilde{u}_{n}\right\|_{\infty}$ with $D$ a constant depending only on the length of [a,b]. So the sequence $\tilde{u}_{n}$ is bounded and then $\left(u_{n}\right)$ is bounded. We reach a contradiction with the fact that $\min u_{n}$ tends to $-\infty$.

Next, let's write the first equation in (2) in the planar system

$$
\begin{aligned}
& u^{\prime}(x)=y(x)-\gamma \tilde{H}(x) \\
& y^{\prime}(x)=-\tilde{g}(x, u(x), \gamma)
\end{aligned}
$$

with $\tilde{g}(x, s, \gamma)=\bar{g}(x, s, \gamma)+c$ and $\tilde{H}(x)=\int_{0}^{x}(h(t)-c) d t$.

Then we have

Lemma 2.2 A positive constant $L$ exists such that any solution $u$ of (3)-(4) such that $u^{*}>L$, fulfills the following conditions.

There exist uniquely determined real numbers $\rho$ and $\bar{\rho}$, with $\alpha_{0}<\rho \leq x^{*} \leq \bar{\rho}<\beta_{0}$ ( where $\alpha_{0}, \beta_{0}$ are as in definition 2.2) such that

1.

$$
\begin{gathered}
y(x)>\|\tilde{H}\|_{\infty} \quad \text { on } \quad\left[\alpha_{0}, \rho[\right. \\
|y(x)| \leq\|\tilde{H}\|_{\infty} \text { on }[\rho, \bar{\rho}] \\
\left.\left.y(x)<-\|\tilde{H}\|_{\infty} \quad \text { on } \quad\right] \bar{\rho}, \beta_{0}\right]
\end{gathered}
$$

2.

$$
u^{*}-L \leq u(x) \leq u^{*} \quad \text { on } \quad[\rho, \bar{\rho}]
$$

Moreover if $\bar{g}(x, ., \gamma)$ is unbounded from above on $\mathbb{R}_{+}$uniformly for $(\gamma \in[0,1]$, and a.e. $x \in[a, b])$, then for any $K$ such that $u^{*}-L \leq K$ we have

3.

$$
\lim _{K \rightarrow+\infty}\left(\alpha_{K}-\alpha_{K-L}\right)=\lim _{K \rightarrow+\infty}\left(\beta_{K-L}-\beta_{K}\right)=0
$$


Remark 2.2 A dual version of lemma (2.2) can be obtained in the case $u$ is a solution with $u_{*}<-L$ and $\bar{g}(x, ., \gamma)$ is unbounded from below on $\mathbb{R}_{-}$uniformly (for $\gamma \in[0,1]$ and a.e. $x \in[a, b])$, by setting $\tilde{g}(x, s, \gamma)=\bar{g}(x, s, \gamma)-c$ and $\tilde{H}(x)=\int_{0}^{x}(h(t)+c) d t$. In this case we have

$$
\alpha_{0}^{\prime}<\rho^{\prime} \leq x_{*} \leq \bar{\rho}^{\prime}<\beta_{0}^{\prime}
$$

such that

$$
\begin{gathered}
y(x)<\|\tilde{H}\|_{\infty} \quad \text { on } \quad\left[\alpha_{0}^{\prime}, \rho^{\prime}[\right. \\
|y(x)| \leq\|\tilde{H}\|_{\infty} \text { on }\left[\rho^{\prime}, \bar{\rho}^{\prime}\right] \\
\left.\left.y(x)>-\|\tilde{H}\|_{\infty} \quad \text { on } \quad\right] \bar{\rho}^{\prime}, \beta_{0}^{\prime}\right] \\
u_{*} \leq u(x) \leq u_{*}+L \quad \text { on } \quad\left[\rho^{\prime}, \bar{\rho}^{\prime}\right]
\end{gathered}
$$

and for any $K$ such that $u_{*}+L \geq K$ we have

$$
\lim _{K \rightarrow+\infty}\left(\alpha_{K-L}^{\prime}-\alpha_{K}^{\prime}\right)=\lim _{K \rightarrow+\infty}\left(\beta_{K}^{\prime}-\beta_{K-L}^{\prime}\right)=0
$$

\section{Proof of lemma 2.2}

From the sign condition $y^{\prime}<0$ on $\left(\alpha_{0}, \beta_{0}\right)$, so $y$ is strictly decreasing and hence

$$
y\left(\alpha_{0}\right)>y\left(x^{*}\right)>y\left(\beta_{0}\right)
$$

Next, as $u^{\prime}\left(x^{*}\right)=0$, we derive that

$$
|y(x)| \leq\|\tilde{H}\|_{\infty}
$$

Integrating the equation in (3) consecutively on $\left[\alpha_{0}, x^{*}\right]$ and $\left[x^{*}, \beta_{0}\right]$, we obtain

$$
\left(x^{*}-\alpha_{0}\right) y\left(\alpha_{0}\right)>\int_{\alpha_{0}}^{x^{*}} y(x) d x=u^{*}+(1-\gamma) \int_{\alpha_{0}}^{x^{*}} \tilde{H}(x) d x \geq u^{*}-(b-a)\|\tilde{H}\|_{\infty}
$$

and

$$
\left(\beta_{0}-x^{*}\right) y\left(\beta_{0}\right)>\int_{x^{*}}^{\beta_{0}} y(x) d x \geq-u^{*}+(b-a)\|\tilde{H}\|_{\infty} .
$$

So for $u^{*}>2(b-a)\|\tilde{H}\|_{\infty}$, one has

$$
y\left(\alpha_{0}\right)>\|\tilde{H}\|_{\infty}, \quad y\left(\beta_{0}\right)<-\|\tilde{H}\|_{\infty}
$$

It appears from (5) and (6) that there exist $\left.\rho \in] \alpha_{0}, x^{*}\right], \quad \bar{\rho} \in\left[x^{*}, \beta_{0}[\right.$ uniquely determined such that

$$
y(x) \leq\|\tilde{H}\|_{\infty}, \quad \text { in }[\rho, \bar{\rho}]
$$

So point (1) of lemma 4.1 is satisfied.

To prove point (2) of the lemma, let's consider two any points $x^{\prime}$ and $x^{\prime \prime}$ in $[\rho, \bar{\rho}]$ and using (3) one has

$$
\left|u\left(x^{\prime \prime}\right)-u\left(x^{\prime}\right)\right| \leq\left|\int_{x^{\prime}}^{x^{\prime \prime}} y(x) d x\right|+(b-a)\|\tilde{H}\|_{\infty} \leq 2(b-a)\|\tilde{H}\|_{\infty}
$$


Taking $x^{\prime}=x^{*}$, one obtains

$$
u^{*}-2(b-a)\|\tilde{H}\|_{\infty} \leq u\left(x^{\prime \prime}\right) \leq u^{*}, \forall x^{\prime \prime} \in[\rho, \bar{\rho}] .
$$

So taking $L=2(b-a)\|\tilde{H}\|_{\infty}$, we have point (2) of the lemma.

For the remaining part we note that since $\bar{g}(x, ., \gamma)$ is unbounded from the above on $\mathbb{R}_{+}$, uniformly for $(\gamma \in[0,1]$, and a.e. $x \in[a, b])$, for any $k>0$, one can choose $K>0$ large enough such that

$$
\bar{g}(x, s, \gamma) \geq k \text { for all } s \in[K-L, K], \gamma \in[0,1] \text {, and a.e. } x \in[a, b] .
$$

with $L=2(b-a)\|\tilde{H}\|_{\infty}$. But for $x \in\left[\alpha_{K-L}, \alpha_{K}\right]$, one has $K-L \leq u(x) \leq K$ and then we have

$$
\tilde{g}(x, u(x), \gamma) \geq k, \quad \forall x \in\left[\alpha_{K-L}, \alpha_{K}\right], \gamma \in[0,1]
$$

Recalling equation (4), we have

$$
y(x)=y\left(\alpha_{K}\right)+\int_{x}^{\alpha_{K}}\left[\tilde{g}(t, u(t), \gamma) d t \text { on }\left[\alpha_{K-L}, \alpha_{K}\right] .\right.
$$

We suppose in the lemma that $u^{*}-L \leq K$, which implies $\alpha_{K} \in[\rho, \bar{\rho}]$.

So using this condition with (7) and (8) we have

$$
y(x) \geq-\|\tilde{H}\|_{\infty}+k\left(\alpha_{K}-x\right)
$$

Taking into account the inequality above in (3), we get

$$
u^{\prime}(x)=y(x)-\gamma \tilde{H}(x) \geq-2\|\tilde{H}\|_{\infty}+k\left(\alpha_{K}-x\right) \quad \text { on }\left[\alpha_{K-L}, \alpha_{K}\right] .
$$

Finally, we derive from an integration of $u^{\prime}$ on $\left[\alpha_{K-L}, \alpha_{K}\right]$ the following inequality

$$
\begin{array}{r}
L=u\left(\alpha_{K}\right)-u\left(\alpha_{K-L}\right) \geq-2\|\tilde{H}\|_{\infty}\left(\alpha_{K}-\alpha_{K-L}\right)+\frac{k}{2}\left(\alpha_{K}-\alpha_{K-L}\right)^{2} \\
\geq-2\|\tilde{H}\|_{\infty}(b-a)+\frac{k}{2}\left(\alpha_{K}-\alpha_{K-L}\right)^{2}
\end{array}
$$

And then

$$
\alpha_{K}-\alpha_{K-L} \geq 2 \sqrt{\frac{L}{k}}
$$

In a similar way we have for interval $\left[\beta_{K}, \beta_{K-L}\right]$ the following

$$
\beta_{K-L}-\beta_{K} \geq 2 \sqrt{\frac{L}{k}}
$$

When $K$ tends to $+\infty, v_{k}$ and then $k$ tends to $+\infty$. Thus point (3) of lemma 2.2 is proved and so is lemma 2.2 .

As a consequence of the third part of the lemma we have the following remark

Remark 2.3 When $K=u^{*}$, then $\alpha_{K}=\beta_{K}=x^{*} \quad \alpha_{K-L}=\rho, \quad \beta_{K-L}=\bar{\rho}$. Consequently, we have $\lim _{u^{*} \rightarrow+\infty}(\bar{\rho}-\rho)=0$.

Now let's go back to our main problem. Our main result is stated as follows. 


\section{Main result}

Theorem 3.1 Suppose that $g$ satisfies the following conditions:

$$
\operatorname{sgn}(s) g(s) \geq-c \quad \text { for a non negative constant } c
$$

Moreover assume

$$
\text { and } \begin{gathered}
\liminf _{s \rightarrow+\infty} \frac{2 G(s)}{s^{2}}<\mu_{+} \quad \liminf _{s \rightarrow-, \infty} \frac{2 G(s)}{s^{2}}<\mu_{-} \\
\limsup _{s \rightarrow \pm \infty} \frac{2 G(s)}{s^{2}}>\lambda_{1} .
\end{gathered}
$$

Then problem (1) is solvable for any $h \in L^{1}(a, b)$.

We denote $G$ the primitive $G(s)=\int_{0}^{s} g(t) d t$.

\section{Proof of the Theorem.}

We consider the following homotopy

$$
\begin{aligned}
-u^{\prime \prime} & =(1-\gamma) \theta u+\gamma g(u)+\gamma h(x) \quad \text { in }(a, b) \\
u & =0 \quad \text { on }\{a, b\}
\end{aligned}
$$

The function $(s, \gamma) \mapsto(1-\gamma) \theta u+\gamma \tilde{g}(u)$ is the particular case of $\bar{g}$ not depending on $x$. It verifies the conditions required on $\bar{g}(x, s, \gamma)$ so that any solutions of (13) satisfy the conclusions of lemmas 2.1 and 2.2 as well. From the assumptions in the theorem we can derive the following lemma.

Lemma 4.1. There exist some sequences $S_{n} \rightarrow+\infty$ and $T_{n} \rightarrow-\infty$ such that if $u$ solves (13) and $u$ changes sign, then either $\max u \neq S_{n}$, or $\min u \neq T_{n}$, for every $n$.

\section{Proof.}

Write $\tilde{G}(s)=\int_{0}^{s}(g(t)+c) d t$ with $c$ as in the sign condition and denote

$$
\begin{aligned}
& \mu=\liminf _{s \rightarrow+\infty} \frac{2 \tilde{G}(s)}{s^{2}} \\
& \nu=\liminf _{s \rightarrow-\infty} \frac{2 \tilde{G}(s)}{s^{2}}
\end{aligned}
$$

Recalling the expression of the first curve in the Fučik spectrum and (10), we observe that $\pi / \sqrt{\mu}+\pi / \sqrt{\nu}>b-a$. Choose $\mu^{\prime}>\mu \quad$ so that we have again

$$
\pi / \sqrt{\mu^{\prime}}+\pi / \sqrt{\nu}>b-a
$$


From the first part of (10) we have

$$
\limsup _{s \rightarrow+\infty}\left(\mu^{\prime} \frac{s^{2}}{2}-\tilde{G}(s)\right)=+\infty
$$

So, there exists an increasing sequence $s_{n} \rightarrow+\infty$ so that for each $n \in \mathbf{N}$

$$
\begin{gathered}
s_{n}>L, \\
\mu^{\prime} \frac{s^{2}}{2}-\tilde{G}(s)<\mu^{\prime} \frac{s_{n}^{2}}{2}-\tilde{G}\left(s_{n}\right)
\end{gathered}
$$

for all $s \in\left[0, s_{n}\left[\right.\right.$ where $L=2(b-a)\|\tilde{H}\|_{\infty}$ with $\tilde{H}(x)=\int_{0}^{x}(h(t)-c) d t$.

Choose $S_{n}$ as a tail sequence of the sequence $s_{n}$ and suppose that with such a $S_{n}$, lemma 4.1 is false. Then, one can find a subsequence of $s_{n}$ still denoted by $s_{n}$, and solutions $u_{n}$ of (13) satisfying $\max u_{n}=s_{n} \rightarrow+\infty$. It results from lemma 2.1 that $m_{n}=\min u_{n} \rightarrow-\infty$. Let's show that such a $u_{n}$ leads to a contradiction.

So, let's write equation (13) in the planar system

$$
\begin{array}{r}
u^{\prime}(x)=y(x)-\gamma \tilde{H}(x) \\
y^{\prime}(x)=(1-\gamma) \theta u(x)-\gamma \tilde{g}(u(x))
\end{array}
$$

and consider the function $T_{\epsilon}$ with $\epsilon= \pm 1$, defined by

$$
T_{\epsilon}(x)=\frac{1}{2}\left(y_{n}(x)+\epsilon\|\tilde{H}\|_{\infty}\right)^{2}+\gamma \tilde{G}\left(u_{n}(x)\right)+(1-\gamma) \theta \frac{u_{n}(x)^{2}}{2} \quad \text { on }[a, b]
$$

One can easily see that

$$
\begin{array}{ll}
T_{-1}^{\prime}(x) \geq 0 & \text { on }\left[\alpha_{0}, \rho\right] \\
T_{1}^{\prime}(x) \leq 0 & \text { on }\left[\bar{\rho}, \beta_{0}\right]
\end{array}
$$

And then

$$
\begin{aligned}
& T_{-1}(x) \leq T(\rho)=\gamma \tilde{G}\left(u_{n}(\rho)+(1-\gamma) \theta \frac{u_{n}(\rho)^{2}}{2} \quad \text { on }\left[\alpha_{0}, \rho\right]\right. \\
& T_{1}(x) \leq T(\bar{\rho})=\gamma \tilde{G}\left(u_{n}(\bar{\rho})+(1-\gamma) \theta \frac{u_{n}(\bar{\rho})^{2}}{2} \quad \text { on }\left[\bar{\rho}, \beta_{0}\right]\right.
\end{aligned}
$$

Consequently we have

$$
\begin{gathered}
T_{-1}(x) \leq \gamma \tilde{G}\left(s_{n}^{2}\right)+(1-\gamma) \theta \frac{s_{n}^{2}}{2} \text { on }\left[\alpha_{0}, \rho\right] \\
T_{1}(x) \leq \gamma \tilde{G}\left(s_{n}^{2}\right)+(1-\gamma) \theta \frac{s_{n}^{2}}{2} \text { on }\left[\bar{\rho}, \beta_{0}\right]
\end{gathered}
$$

Taking into account (15) and choosing $\theta$ such that $\lambda_{1}<\theta<\mu^{\prime}$, we get the following

$$
\begin{aligned}
& \frac{u_{n}^{\prime}(x) d x}{2\|\tilde{H}\|_{\infty}+\left[\mu^{\prime}\left(s_{n}^{2}-u_{n}^{2}(x)\right)\right]^{1 / 2}} \leq 1 \text { on }\left[\alpha_{0}, \rho\right] \\
& \frac{-u_{n}^{\prime}(x) d x}{2\|\tilde{H}\|_{\infty}+\left[\mu^{\prime}\left(s_{n}^{2}-u_{n}^{2}(x)\right)\right]^{1 / 2}} \leq 1 \text { on }\left[\bar{\rho}, \beta_{0}\right]
\end{aligned}
$$


and then

$$
\left(\beta_{0}-\alpha_{0}\right) \geq\left(\beta_{0}-\bar{\rho}\right)+\left(\rho-\alpha_{0}\right) \geq \frac{2}{\sqrt{\mu^{\prime}}} \int_{0}^{s_{n}-L} \frac{d \xi}{K+\left(s_{n}^{2}-\xi^{2}\right)^{1 / 2}}
$$

with $K=2\|\tilde{H}\|_{\infty} \mu^{\prime-1 / 2}$. And finally

$$
\liminf _{n \rightarrow \infty}\left(\beta_{0}-\alpha_{0}\right) \geq \pi / \sqrt{\mu^{\prime}}
$$

Next, we will give an estimate of the length $\left(\beta_{0}^{\prime}-\alpha_{0}^{\prime}\right)$. So let's write here $\tilde{g}(s)=g(s)-c$ and $\tilde{G}(s)=\int_{0}^{s} \tilde{g}(t) d t$. Arguing as in (lemma 2, [4]), we can choose $m_{n}<-2 L$ and readily establish the following

$$
m_{n} \leq u_{n}\left(\rho^{\prime}\right), u_{n}\left(\overline{\rho^{\prime}}\right) \leq m_{n}+L
$$

Moreover, by performing $T_{-1}^{\prime}$ and $T_{1}^{\prime}$ respectively on $\left[\alpha_{0}^{\prime}, \rho^{\prime}\right]$ and $\left[\bar{\rho}^{\prime}, \beta_{0}^{\prime}\right]$, we have

$$
\begin{array}{ll}
T_{-1}^{\prime}(x) \leq 0 & \text { on }\left[\alpha_{0}^{\prime}, \rho^{\prime}\right] \\
T_{1}^{\prime}(x) \geq 0 & \text { on }\left[\bar{\rho}^{\prime}, \beta_{0}^{\prime}\right]
\end{array}
$$

and then

$$
\begin{aligned}
& T_{-1}(x) \leq T\left(\rho^{\prime}\right)=\gamma \tilde{G}\left(u_{n}\left(\rho^{\prime}\right)+(1-\gamma) \theta \frac{u_{n}\left(\rho^{\prime}\right)^{2}}{2} \quad \text { on }\left[\alpha_{0}^{\prime}, \rho^{\prime}\right]\right. \\
& T_{1}(x) \leq T\left(\bar{\rho}^{\prime}\right)=\gamma \tilde{G}\left(u_{n}\left(\bar{\rho}^{\prime}\right)+(1-\gamma) \theta \frac{u_{n}\left(\bar{\rho}^{\prime}\right)^{2}}{2} \quad \text { on }\left[\bar{\rho}^{\prime}, \beta_{0}^{\prime}\right]\right.
\end{aligned}
$$

Using the fact that $\tilde{G}$ is decreasing on $\mathbb{R}_{-}$, one has

$$
\begin{array}{rrr}
-u_{n}^{\prime}(x) & \leq 2\|\tilde{\tilde{H}}\|_{\infty}+\left[R_{\gamma}\left(m_{n}\right)-R_{\gamma}\left(u_{n}(x)\right)\right] & \text { on }\left[\alpha_{0}^{\prime}, \rho^{\prime}\right] \\
u_{n}^{\prime}(x) & \leq 2\|\tilde{\tilde{H}}\|_{\infty}+\left[R_{\gamma}\left(m_{n}\right)-R_{\gamma}\left(u_{n}(x)\right)\right] & \text { on }\left[\bar{\rho}^{\prime}, \beta_{0}^{\prime}\right]
\end{array}
$$

where $R_{\gamma}(x)=\int_{0}^{x} r_{\gamma}(s) d s$ and $r_{\gamma}(s)=\gamma \tilde{g}(s)+(1-\gamma) \theta s$.

And then we get

$$
\left(\beta_{0}^{\prime}-\alpha_{0}^{\prime}\right) \geq \sqrt{2} \int_{m_{n}}^{0} \frac{d \xi}{\sqrt{2}\|\tilde{\tilde{H}}\|_{\infty}+\left[R_{\gamma_{n}}\left(m_{n}\right)-R_{\gamma_{n}}(\xi)\right]^{1 / 2}}
$$

To go further in the estimation of the length $\left(\beta_{0}^{\prime}-\alpha_{0}^{\prime}\right)$ let's set the following. We define $T_{\gamma}(x)$ and $\tau_{\gamma}(x)$ by

$$
T_{\gamma}(x)=\sqrt{2} \int_{0}^{x} \frac{d \xi}{\sqrt{2}|| \tilde{\tilde{H}} \|_{\infty}+\left[R_{\gamma}\left(m_{n}\right)-R_{\gamma}(\xi)\right]^{1 / 2}}
$$

and

$$
\tau_{\gamma}(x)=\sqrt{2} \int_{0}^{x} \frac{d \xi}{\left[R_{\gamma}\left(m_{n}\right)-R_{\gamma}(\xi)\right]^{1 / 2}}
$$

when $x>0$ 
and

$$
T_{\gamma}(x)=\sqrt{2} \int_{x}^{0} \frac{d \xi}{\sqrt{2}|| \tilde{\tilde{H}} \|_{\infty}+\left[R_{\gamma}\left(m_{n}\right)-R_{\gamma}(\xi)\right]^{1 / 2}}
$$

and

$$
\tau_{\gamma}(x)=\sqrt{2} \int_{x}^{0} \frac{d \xi}{\left[R_{\gamma}(x)-R_{\gamma}(\xi)\right]^{1 / 2}}
$$

when $x<0$

The following assertion is verified

\section{Claim 1 .}

$$
\lim _{x \rightarrow \pm \infty}\left[\tau_{\gamma}(x)-T_{\gamma}(x)\right]=0 \text { uniformly with respect to } \gamma \in[0,1]
$$

\section{Proof.}

We refer to the proof of claim 1 in [4].

Next, let's define for $\eta>0$ and $x$ real number the set $A_{x}^{\eta}$ as follows

$$
A_{x}^{\eta}=\left\{\xi \in[0, x], R_{\gamma}(x)-R_{\gamma}(\xi) \leq \frac{1}{2} \eta\left(x^{2}-\xi^{2}\right), \mathrm{x} \geq 0\right\}
$$

or

$$
A_{x}^{\eta}=\left\{\xi \in[x, 0], R_{\gamma}(x)-R_{\gamma}(\xi) \leq \frac{1}{2} \eta\left(x^{2}-\xi^{2}\right), \mathrm{x} \leq 0\right\}
$$

We denote by $\left|A_{x}^{\eta}\right|$ the Lebesgue measure of $A_{x}^{\eta}$. We have the two following claims.

Claim 2. Assume $\limsup _{x \rightarrow \pm \infty}\left(\left|A_{x}^{\eta}\right| / x\right) \geq \rho^{ \pm}\left(\right.$resp. $\left.\liminf _{x \rightarrow \pm \infty}\left(\left|A_{x}^{\eta}\right| / x\right) \geq \rho_{ \pm}\right)$

Then

$$
\limsup _{x \rightarrow \pm \infty} \tau_{\gamma}(x) \geq \frac{2}{\sqrt{\eta}} \arcsin \rho^{ \pm}\left(\operatorname{resp} .\left(\liminf _{x \rightarrow \pm \infty} \tau_{\gamma}(x)\right) \geq \frac{2}{\sqrt{\eta}} \arcsin \rho_{ \pm}\right) .
$$

uniformly with respect to $\gamma$

\section{Proof.}

We will give the proof only in case where $x \rightarrow-\infty$. The case $x \rightarrow+\infty$ follows in a similar way. For $x \leq 0$ and sufficiently large we have

$$
\begin{aligned}
\tau_{\gamma}(x) & =\sqrt{2} \int_{x}^{0} \frac{d \xi}{\left[R_{\gamma}(x)-R_{\gamma}(\xi)\right]^{1 / 2}} \\
& \geq \frac{2}{\sqrt{\eta}} \int_{A_{x}^{\eta}} \frac{d \xi}{\left[x^{2}-\xi^{2}\right]^{1 / 2}}
\end{aligned}
$$

Now, since $x \longmapsto\left(x^{2}-s^{2}\right)^{-} 1 / 2$ is an decreasing function on $[x, 0]$ we have (see [Fo-Za],[DF-Go])

$$
\tau_{\gamma}(x) \geq \frac{2}{\sqrt{\eta}} \int_{\left|A_{x}^{\eta}\right|}^{0} \frac{d \xi}{\left[x^{2}-\xi^{2}\right]^{1 / 2}}=\frac{2}{\sqrt{\eta}} \arcsin \left(\left|A_{x}^{\eta}\right| / x\right)
$$

So, $\limsup _{x \rightarrow-\infty} \tau_{\gamma}(x) \geq \frac{2}{\sqrt{\eta}} \arcsin \rho^{-}$resp. $\left(\liminf _{x \rightarrow-\infty} \tau_{\gamma}(x) \geq \frac{2}{\sqrt{\eta}} \arcsin \rho_{-}\right)$. 
Claim $3 . \limsup _{x \rightarrow-\infty} \tau_{\gamma}(x) \geq \pi / \sqrt{\nu}$ uniformly with respect to $\gamma \in[0,1]$

\section{Proof .}

We observe that

$\liminf _{x \rightarrow-\infty} 2 R_{\gamma}(x) / x^{2} \leq \nu$ for all $\gamma \in[0,1]$.

Take $\eta=\nu+\epsilon$, with $\epsilon>0$, then

$$
\limsup _{x \rightarrow-\infty}\left(\frac{1}{2} \eta x^{2}-R_{\gamma}(x)\right)=+\infty
$$

and so there exists, a decreasing sequence $x_{n} \rightarrow-\infty$ so that $\left|A_{x_{n}}^{\eta}\right|=x_{n}$. It results that $\limsup _{x \rightarrow-\infty}\left(\left|A_{x_{n}}^{\eta}\right| / x_{n}\right)=1$ and then by claim 2

$$
\limsup _{x \rightarrow-\infty} \tau_{\gamma}(x) \geq \pi / \sqrt{\eta}
$$

As $\epsilon \rightarrow 0$, we reach the result.

We are now ready to complete the proof of lemma 4.1. From claim 3, there is a sequence $t_{n} \rightarrow+\infty$ such that

$$
\lim _{n \rightarrow+\infty} \tau_{\gamma}\left(t_{n}\right) \geq \pi / \sqrt{\nu}
$$

for all $\gamma \in[0,1]$. Take $T_{n}$ as a tail sequence of the sequence $t_{n}$. Since we suppose that the assertions in lemma 4.1 are false, for a subsequence of $m_{n}$, we have $m_{n}=t_{n}$ for every $n$ and then, by claim 1 we have

$$
T_{\gamma}\left(t_{n}\right) \geq \tau_{\gamma}\left(t_{n}\right)-\epsilon / 2 .
$$

It follows that

$$
\begin{aligned}
b-a & \geq\left(\beta_{0}-\alpha_{0}\right)+\left(\beta_{0}^{\prime}-\alpha_{0}^{\prime}\right) \geq\left(\beta_{0}-\alpha_{0}\right)+T_{\gamma}\left(t_{n}\right) \\
& \left.\geq \beta_{0}-\alpha_{0}\right)+\tau_{\gamma}\left(t_{n}\right)-\epsilon \geq \pi / \sqrt{\mu^{\prime}}+\pi / \sqrt{\nu}>b-a .
\end{aligned}
$$

Contradiction. So lemma 4.1 is proved, and then either $\max u \neq S_{n}$ or $\min u \neq T_{n}$ for any solution of (13) which changes sign.

Next we state the following lemma

Lemma 4.2 There exists a sequence $K_{n}$ tending to $+\infty$ such that if $u$ is a nonnegative solution of (13) for some $\gamma \in[0,1]$, then $u^{*}<K_{n}$ for $n$ large enough.

Remark 4.1 A dual version of the claim above can be obtained in case $u$ is non-positive. In this case there is a sequence $K_{n}^{\prime} \rightarrow-\infty$ such that $u_{*}>K_{n}^{\prime}$ for $n$ large enough.

Proof of lemma 4.2. The proof uses the first half of assumption (12), say

$$
\limsup _{s \rightarrow \pm \infty} \frac{2 G(s)}{s^{2}}>\lambda_{1} .
$$


The second half is needed when dealing with the case $u$ non-positive.

Write $\lambda=\limsup _{s \rightarrow+\infty} \frac{2 \tilde{G}(s)}{s^{2}}$ where $\tilde{G}$ is as in lemma 4.1. From assumption (12) of the theorem it is clear that $\lambda>\lambda_{1}$. Choose $\mu$ such that $\lambda_{1}<\mu<\lambda$, then we have

$$
\limsup _{s \rightarrow+\infty}\left(2 \tilde{G}(s)-\mu s^{2}\right)=+\infty .
$$

So there exists an increasing sequence $k_{n} \rightarrow+\infty, k_{n}>L$ such that

$$
\mu \frac{s^{2}}{2}-\tilde{G}(s)<\mu \frac{k_{n}^{2}}{2}-\tilde{G}\left(k_{n}\right)
$$

for all $s \in\left[0, k_{n}\right.$ [. Let's show that $K_{n}$ taken equal to a $k_{n}$ for $n$ large enough is convenient. On the contrary, one can find a sequence of non-negative solutions $\left(u_{n}\right)$ and some corresponding $(\gamma) \in[0,1]$, and a subsequence of $k_{n}$ still denoted by $k_{n}$ such that the following is satisfies

$$
u_{n}(x)=k_{n} \text { for some } x \in[a, b], \quad k_{n} \rightarrow+\infty .
$$

We further point out that (20) is absurd. So, let us consider the function $T_{\epsilon}$ as defined in lemma 4.1.

$$
T_{1}^{\prime}(x) \leq 0 \text { on }[a, b] \text { and } T_{-1}^{\prime}(x) \geq 0 \text { on }[a, b] .
$$

Considering $T_{1}$ on $\left[a, \alpha_{k_{n}}\right], T_{-1}$ on $\left[\beta_{k_{n}}, b\right]$ where $\alpha_{k_{n}}, \beta_{k_{n}}$ are as in definition 2, and taking into account (19) we have the following

$$
\begin{aligned}
& \frac{1}{2}\left(y(x)+\|\tilde{H}\|_{\infty}\right)^{2} \geq \frac{\mu}{2}\left(k_{n}^{2}-u^{2}(x)\right) \text { on }\left[a, \alpha_{k_{n}}[\right. \\
& \left.\left.\frac{1}{2}\left(y(x)+\|\tilde{H}\|_{\infty}\right)^{2} \geq \frac{\mu}{2}\left(k_{n}^{2}-u^{2}(x)\right) \text { on }\right] \beta_{k_{n}}, b\right]
\end{aligned}
$$

And then

$$
\begin{aligned}
u^{\prime}(x) & \geq-2\|\tilde{H}\|_{\infty}+\quad\left[\mu\left(k_{n}^{2}-u^{2}(x)\right)\right]^{1 / 2} \text { on }\left[a, \alpha_{k_{n}}[\right. \\
-u^{\prime}(x) & \left.\left.\geq-2\|\tilde{H}\|_{\infty}+\quad\left[\mu\left(k_{n}^{2}-u^{2}(x)\right)\right]^{1 / 2} \text { on }\right] \beta_{k_{n}}, b\right]
\end{aligned}
$$

Let us show that the right-hand side term of the inequalities above are strictly positive on $\left[a, \alpha_{k_{n}-L}\right] \cup\left[\beta_{k_{n}-L}, b\right]$.

Indeed, for $x \in\left[a, \alpha_{k_{n}-L}\right] \cup\left[\beta_{k_{n}-L}, b\right]$, we have $0 \leq u(x) \leq k_{n}-L$ and then $L^{2} \leq k_{n}^{2}-u^{2}(x)$ Thus

$$
-2|| \tilde{H}\left\|_{\infty}+\left[\mu\left(k_{n}^{2}-u^{2}(x)\right)\right]^{1 / 2} \geq-2|| \tilde{H}\right\|_{\infty}+\mu^{1 / 2} L>-2\|\tilde{H}\|_{\infty}+\lambda_{1}{ }^{1 / 2}
$$

Since $L=2(b-a)\|\tilde{H}\|_{\infty}$ we get

$$
-2\|\tilde{H}\|_{\infty}+\left[\mu\left(k_{n}^{2}-u^{2}(x)\right)\right]^{1 / 2}>2\|\tilde{H}\|_{\infty}(\pi-1)>0
$$

Hence one can write

$$
1 \leq \frac{u_{n}^{\prime}(x) d x}{-2\|\tilde{H}\|_{\infty}+\left[\mu\left(k_{n}^{2}-u_{n}^{2}(x)\right)\right]^{1 / 2}} \quad \text { on }\left[a, \alpha_{k_{n}-L}\right]
$$




$$
1 \leq \frac{-u_{n}^{\prime}(x) d x}{-2\|\tilde{H}\|_{\infty}+\left[\mu\left(k_{n}^{2}-u_{n}^{2}(x)\right)\right]^{1 / 2}} \quad \text { on }\left[\beta_{k_{n}-L}, b\right]
$$

Integrating (21) on $\left[a, \alpha_{k_{n}-L}\right]$ and $(22)$ on $\left[\beta_{k_{n}-L}, b\right]$, we get

$$
\left(b-\beta_{k_{n}-L}\right)+\left(\alpha_{k_{n}-L}-a\right) \leq \tau(n)=\frac{2}{\sqrt{\mu}} \int_{0}^{k_{n}-L} \frac{d \xi}{K+\left(k_{n}^{2}-\xi^{2}\right)^{1 / 2}}
$$

with $K=-2\|\tilde{H}\|_{\infty} \mu^{-1 / 2}$ and

$$
\lim _{n \rightarrow+\infty} \tau(n)=\frac{\pi}{\sqrt{\mu}}<b-a
$$

But

$$
b-a=\tau(n)+\left(\beta_{k_{n}-L}-\beta_{k_{n}}\right)+\left(\beta_{k_{n}}-\alpha_{k_{n}}\right)+\left(\alpha_{k_{n}}-\alpha_{k_{n}-L}\right)
$$

Since limits in assumptions (12) are positive, $g(s)$ is unbounded from the above on $\mathbb{R}_{+}$( and from below on $\left.\mathbb{R}_{-}\right)$. It results that $(1-\gamma) \theta u+\gamma g(s)$ is unbounded uniformly for $\gamma \in[0,1]$, so lemma 2.1 and remark 2.3 yield

$$
\lim _{n \rightarrow+\infty}\left(\beta_{k_{n}-L}-\beta_{k_{n}}\right)=\lim _{n \rightarrow+\infty}\left(\alpha_{k_{n}}-\alpha_{k_{n}-L}\right)=0
$$

and

$$
\lim _{n \rightarrow+\infty}\left(\beta_{k_{n}}-\alpha_{k_{n}}\right) \leq \lim _{u_{n}^{*} \rightarrow+\infty}(\bar{\rho}-\rho)=0
$$

So, using (23) we get

$$
b-a \leq \lim _{n \rightarrow+\infty} \tau(n)=\frac{\pi}{\sqrt{\mu}}<b-a .
$$

This is a contradiction and then lemma 4.2 is proved.

The proof of the dual version of lemma 4.2 in the case of non- positive solution follows similarly.

We are now ready to construct open bounded sets on which invariance of topological degree argument will be used to conclude the proof of the theorem. Indeed, choose $K_{n}$ as in lemma 4.2 and $K_{n}^{\prime}$ as in remark 4.1. Choose $S_{\bar{n}}$ and $T_{\bar{n}}$ as in lemma 4.1 such that $S_{\bar{n}}>K_{n}$ and $T_{\bar{n}}<K_{n}^{\prime}$. For the $S_{\bar{n}}, T_{\bar{n}}$ so chosen, lemma 2.1 provided respectively some corresponding positive real numbers $M$ and $M^{\prime}$. Take $-R=\min \left(K_{n}^{\prime},-M\right)$ and $R^{\prime}=\max \left(M^{\prime}, K_{n}\right)$. Next, we set

$$
\begin{aligned}
& \Omega_{1}=\left\{u \in C[a, b],-R<u(x)<S_{\bar{n}}, \forall x \in[a, b]\right\} \\
& \Omega_{2}=\left\{u \in C[a, b], T_{\bar{n}}<u(x)<R^{\prime}, \forall x \in[a, b]\right\} .
\end{aligned}
$$

Let us show that for any solutions $u$ of $(13), \Omega_{1}$ is a convenient set on which invariance of topological degree is verified if $u^{*} \neq S_{\bar{n}}$, and if not,$\Omega_{2}$ is the convenient set. So, suppose first that $u$ is a solution which changes sign. Then if $u^{*} \neq S_{\bar{n}}$, from lemma $2.1, u_{*} \neq-R$, and consequently $\Omega_{1}$ is a convenient set. If $u^{*}=S_{\bar{n}}$, lemma 2.2 yields $u_{*} \neq T_{\bar{n}}$ and then lemma 2.1 provided with $R^{\prime}$ such that $u^{*} \neq R^{\prime}$. So $\Omega_{2}$ is a convenient set. 
Now let's deal with the cases where $u$ has a definite sign. Suppose first that $u$ is non-negative. Suppose in this case that $u^{*}=S_{\bar{n}}$, then $u^{*}>K_{n}$ and then we get a contradiction with lemma 4.2. Hence $u^{*} \neq S_{\bar{n}}$ and from lemma $2.1 u_{*} \neq-R$. Next, suppose $u^{*}=R^{\prime}$, one has again $u^{*}>K_{n}$. This is again a contradiction. So in the non negative case $\Omega_{1}$ and $\Omega_{2}$ are convenient sets. A similar argument for the case $u$ non positive leads to the same conclusion as above. Thus, in general problem (1) is solvable either in $\Omega_{1}$ or in $\Omega_{2}$.

\section{Example}

Modifying slightly the example of the nonlinearity given in [2], we get an example of function $g$ for which our result applies. We begin as in [2] by considering $\left(a_{n}\right),\left(b_{n}\right)$ two increasing sequences of positives real numbers defined by the recurrence relations

$$
a_{1}=2, \quad a_{n}=n^{4} a_{n-1}^{2}, \quad b_{n}=n a_{n}^{2} .
$$

Next, we define a function $\tilde{g}$ from: $\mathbb{R}_{+} \rightarrow \mathbb{R}_{+}$to be the piecewise linear continuous function such that

$$
\tilde{g}(0)=0, \tilde{g}\left(a_{n}\right)=\sum_{i=1}^{n} b_{i} \text {, and } \tilde{g}\left(a_{n}-2\right)=\tilde{g}\left(a_{n-2}\right) \text {, for } n>2 .
$$

One can easily check that $\tilde{g}\left(a_{n}\right)>a_{n}$ and $a_{n}-2>\tilde{g}\left(a_{n-2}\right)$. So $\tilde{g}$ is far to be monotone. We extend $\tilde{g}$ over all $\mathbb{R}$ as follows: $\hat{g}(s)=-\tilde{g}(-s)$ for $s \leq 0$.

We observe that $\hat{g}$ is unbounded from above on $\mathbb{R}_{+}$, unbounded from below on $\mathbb{R}_{-}$. Furthermore the following properties are satisfied.

$$
\begin{array}{r}
\liminf _{s \rightarrow \pm \infty} \frac{\hat{g}(s)}{s}=0, \quad \limsup _{s \rightarrow \pm \infty} \frac{\hat{g}(s)}{s}=+\infty \\
\liminf _{s \rightarrow \pm \infty} \frac{2 \hat{G}(s)}{s^{2}}=0, \quad \limsup _{s \rightarrow \pm \infty} \frac{2 \hat{G}(s)}{s^{2}}=+\infty
\end{array}
$$

To achieve our construction, we define $g: \mathbb{R} \rightarrow \mathbb{R}$ by

$$
g(s)=\alpha s^{+}-\beta s^{-}+\hat{g}(s) \text { with } \lambda_{1}<\alpha<\mu, \quad \lambda_{1}<\beta<\mu_{-} .
$$

For such a $g$ the requirements of our theorem are satisfied, namely

$$
\liminf _{s \rightarrow \pm \infty} \frac{2 G(s)}{s^{2}}<\mu_{ \pm} \text {and } \limsup _{s \rightarrow \pm \infty} \frac{2 G(s)}{s^{2}}>\lambda_{1}
$$

and $g$ satisfies the sign condition as well. Accordingly, problem (1)-(2) with $g$ as above admits a solution. As far as we know, no one of the results in [3] and [5] can be applied to this example.

Acknowledgments. This work was done while the author was visiting The Abdus Salam ICTP. The author thanks the Director of the Centre, Professor M. A. Virasoro, UNESCO and IAEA for hospitality. The author thanks the CDE-IMU for travel support. 


\section{References}

[1] D.DE FIGUEIREDO,J.P.GOSSEZ, Nonresonance below the first eigenvalue for a semilinear elliptic problem, Mathematische Annalen,281(1988),589-610.

[2] M.L.C. FERNANDES, P. OMARI, and F. ZANOLIN, On the solvability of a semilinear two-point BVP around the first eigenvalue, Differential and Integral Equations, 2 (1989),6379 .

[3] J.P.GOSSEZ and P. OMARI, On a semilinear elliptic Neumann problem with asymmetric nonlinearities, Transactions of the American Mathematical Society. 347 (1995), 2553-2562.

[4] A.MARCOS, Existence of solution for a semilinear two-point boundary value problem, Nonlinear Analysis T.M.A.(in press)

[5] F.I. NJOKU, F. ZANOLIN, On the solvability of a nonlinear two-point BVP between the first two eigenvalues, Differential and Integral Equations, 3 (1990), 571-588. 